\title{
The Smiley Prize
}

The Smiley Prize, established in honour of Donald V. Smiley (19211990), internationally renowned scholar of Canadian politics and a former President of the Canadian Political Science Association, will be awarded in 1996. The prize, to which is attached a monetary award in the amount of $\$ 750$, is awarded biennially to the author or authors of the best book published in English or French in a field relating to the study of government and politics in Canada in the preceding two-year period. The award-winning book may be single-authored or multi-authored and will be selected by the Board of Directors of the Canadian Political Science Association, upon the recommendation of the Prize Jury. No textbooks, edited texts or collections of essays will be considered. The Smiley Prize is sponsored by McGraw-Hill Ryerson Publishers. Individuals who have published a book in 1994 or 1995 which is eligible for the prize should ensure that their publishers forward one copy of the book by December 10, 1995, to each of: (1) Smiley Prize Jury, The Canadian Political Science Association, Suite 205, 1 Stewart Street, Ottawa, Ontario K1N 6H7; (2) Smiley Prize Jury, Professor Herman Bakvis, Department of Political Science, Dalhousie University, Halifax, Nova Scotia B3H 4H6; (3) Smiley Prize Jury, Professor Elisabeth Gidengil, Department of Political Science, McGill University, 855 Sherbrooke West, Montréal, Québec H3A 2T5; and (4) Smiley Prize Jury, Professor Stephen McBride, Department of Political Science, Simon Fraser University, Burnaby, British Columbia V5A 1 S6.

\section{Le Prix Smiley}

Le Prix Smiley a été créé en hommage à Donald V. Smiley (19211990), le grand spécialiste de la politique canadienne dont la réputation dépassait nos frontières et un ancien président de l'Association canadienne de science politique. L'Association décernera le Prix Smiley, d'une valeur de 750 \$, en 1996 à l'auteur, l'auteure ou aux auteurs du meilleur livre publié en français ou en anglais sur un sujet traitant de la politique ou du gouvernement au Canada au cours de la période correspondante de deux ans. Le Conseil d'administration de l'Association désignera le lauréat, la lauréate ou les lauréats à la suite d'une recommandation du Jury du Prix Smiley. Les livres avec directeur ou codirecteurs, les manuels de classe et les collections d'essais ne seront pas éligibles. Le Prix Smiley est subventionné à tous les deux ans par les éditeurs McGraw-Hill Ryerson. Les personnes qui auraient publié un ouvrage en 1994 ou en 1995 selon les conditions du concours devront, pour être éligibles, demander à leurs éditeurs de faire parvenir un exem- 
plaire de leur ouvrage, avant le 10 décembre 1995 au : (1) Jury du Prix Smiley, Association canadienne de science politique, Bureau 205, 1, rue Stewart, Ottawa, Ontario K1N 6H7; (2) Jury du Prix Smiley, Professor Herman Bakvis, Department of Political Science, Dalhousie University, Halifax, Nova Scotia B3H 4H6; (3) Jury du Prix Smiley, Professor Elisabeth Gidengil, Department of Political Science, McGill University, 855 Sherbrooke West, Montréal, Québec H3A 2T5; et (4) Jury du Prix Smiley, Professor Stephen McBride, Department of Political Science, Simon Fraser University, Burnaby, British Columbia V5A 1 S6. 